\title{
CARBON AND NITROGEN ABUNDANCES IN EARLY-TYPE GALAXIES
}

\author{
E. Toloba ${ }^{1}$, P. SÁnchez-Blázquez ${ }^{2}$, J. Gorgas ${ }^{1}$, and B. K. Gibson ${ }^{2}$ \\ ${ }^{1}$ Universidad Complutense de Madrid, 28040, Madrid, Spain \\ ${ }^{2}$ Centre for Astrophysics, University of Central Lancashire, Preston PR1 2HE, UK \\ Received 2008 October 10; accepted 2008 November 19; published 2009 January 12
}

\begin{abstract}
For the first time, we undertake a systematic examination of the nitrogen abundances using the NH3360 feature for a sample of 35 early-type galaxies spanning a range of masses and local environment. The nitrogen-sensitive molecular feature at $3360 \AA$ Å has been employed in conjunction with a suite of atomic- and molecular-sensitive indices to provide unique and definitive constraints on the chemical content of these systems. By employing NH3360, we are now able to break the carbon, nitrogen, and oxygen degeneracies inherent to the use of the cyanogen $(\mathrm{CN})$ index. We demonstrate that the NH3360 feature shows little dependency upon the velocity dispersion (our proxy for mass) of the galaxies, contrary to what is seen for carbon- and magnesium-sensitive indices. At face value, these results are at odds with conclusions drawn previously using indices sensitive to both carbon and nitrogen, such as CN. With the aid of stellar population models, we find that the $\mathrm{N} / \mathrm{Fe}$ ratios in these galaxies are consistent with being mildly enhanced with respect to the solar ratio. We also explore the dependence of these findings upon environment, by analyzing the co-added spectra of galaxies in the field and the Coma cluster. We confirm the previously found differences in carbon abundances between galaxies in low- and high-density environments, while showing that these differences do not seem to exist for nitrogen. We discuss the implications of these findings for the derivation of the star-formation histories in early-type galaxies, and for the origin of carbon and nitrogen themselves.
\end{abstract}

Key words: galaxies: abundances - galaxies: elliptical and lenticular, cD - galaxies: evolution - galaxies:

formation - galaxies: stellar content

\section{INTRODUCTION}

Uncovering the star-formation histories of early-type galaxies has proved to be a challenging endeavor for several decades now. Perhaps the most common approach to date has been via the comparison of Lick/IDS spectral indices, each with varying degrees of sensitivity to age and metallicity (Worthey 1994). Unfortunately, the lack of indices which are purely sensitive to age and/or metallicity has limited the uniqueness of the claimed results. Despite a rich literature, there remains fundamental disagreement on the magnitude and even veracity of important trends, including those linking age, metallicity, and relative elemental abundances, with mass and environment. A further limitation to this technique is its sensitivity to the youngest stellar component in a given galaxy, as opposed to the more representative underlying population.

An alternative approach, and one employed successfully for our own Galaxy, is to use our knowledge of galactic chemical evolution to inform our derivation of star-formation histories. In massive early-type galaxies, O'Connell (1976) first noticed that $\mathrm{Mg}$ abundance was enhanced relative to $\mathrm{Fe}$; other works confirmed this by comparing absorption line indices with isochrone-based stellar population models. Because $\mathrm{Mg}$ is predominantly produced in Type II supernovae, while Fe production is dominated by Type Ia supernovae, this enhancement is often interpreted as the result of very short timescales for the formation of stars in these galaxies. Apart from $\mathrm{Mg}$, other elements (e.g. C, N, Na) have been claimed to be enhanced in large ellipticals (Worthey 1998; Burstein 2003; Kelson et al. 2006; Sánchez-Blázquez et al. 2003, 2006 (SB03 and SB06a hereafter); Schiavon 2007; Graves \& Schiavon 2008) and to be strongly correlated with their velocity dispersions (although see Kelson et al. 2006; Clemens et al. 2006 for an alternate perspective regarding $\mathrm{Mg}$ and $\mathrm{C}$ ).
Claims have been made for a strong $\mathrm{N} / \mathrm{Fe}$ correlation with mass (SB06a; Kelson et al. 2006; Schiavon 2007; Graves \& Schiavon 2008). Nitrogen is produced during hydrogen burning via the $\mathrm{CNO}$ and $\mathrm{CN}$ cycles, and it is created as both primary and secondary element. In primary nucleosynthesis, $\mathrm{N}$ is produced at the same time as $\mathrm{C}$ and $\mathrm{O}$, and it is independent of metallicity, while in secondary production nitrogen is synthesized from the carbon and oxygen already present in the star, and its abundance is therefore proportional to the heavy-element abundance. The correlation of $\mathrm{N} / \mathrm{Fe}$ with mass has been interpreted as a correlation between $\mathrm{N} / \mathrm{Fe}$ and metallicity (through the massmetallicity relation) and has led the authors above to conclude that most of the $\mathrm{N}$ produced in ellipticals is of secondary origin. To derive nitrogen abundances, the CN-band at $4175 \AA$ has typically been used; however, disentangling the effects of $\mathrm{C}, \mathrm{N}$, and $\mathrm{O}$ using the CN-band is difficult (Burstein 2003). Direct observation of the NH feature at $3360 \AA$ offers a more direct and robust estimator as the $\mathrm{NH}$ feature is insensitive to C and O (Sneden 1973; Norris et al. 2002), and it is directly measuring N abundances (Bessell \& Norris 1982; Tomkin \& Lambert 1984). In addition, the near-UV light is produced primarily by dwarf stars (Davidge \& Clark 1994), while the optical cyanogen indices result from a more complex mix of both dwarf and giant star light. This avoids the variation in the stellar atmospheric abundances due to mixing during the first dredge-up.

There are fewer than 15 early-type galaxies with published values of NH3360 in the literature (Ponder et al. 1998; Boulade et al. 1988; Davidge \& Clark 1994), due in large part to the relative insensitivity of detectors in the near-UV. We present here observations of the $\mathrm{NH}$ feature in a sample of 35 early-type galaxies in the field, Virgo, and Coma clusters. Contrary to the results obtained with the $\mathrm{CN}$, we found that the relation between $\mathrm{N}$ and $\sigma$ is flat. 
Table 1

Index Definitions

\begin{tabular}{lcccc}
\hline \hline Name & Blue Passband $(\AA)$ & Index Passband $(\AA)$ & Red Passband $(\AA)$ & Reference \\
\hline NH33960 & $3320-3350$ & $3350-3400$ & $3415-3435$ & Davidge \& Clark (1994) \\
CNO3862 & $3768.1-3812.3$ & $3840.3-3883.4$ & $3896.4-3916.2$ & SWB05 \\
CNO4175 & $4082.5-4123.3$ & $4129.4-4219.8$ & $4243.3-4284.2$ & SWB05 \\
CO4685 & $4557.3-4589.5$ & $4626.4-4743.3$ & $4805.1-4835.3$ & SWB05 \\
Mgb & $5142.6-5161.4$ & $5160.1-5192.6$ & $5191.4-5206.4$ & Burstein et al. (1984) \\
\hline
\end{tabular}

\section{OBSERVATIONS AND DATA REDUCTION}

Long-slit spectroscopic data for 35 ellipticals were obtained at the $4.2 \mathrm{~m}$ William Herschel Telescope at Roque de los Muchachos Observatory, using the ISIS spectrograph. The instrumental configuration provided spectral coverage from 3140 to $4040 \AA$ in the blue arm with a spectral resolution of $2.3 \AA$ (FWHM) and a typical signal to noise per $\AA$ of 40 . Our sample is a subset of those presented in SB06a, allowing us to supplement our near-UV data with the associated optical indices (3500-5250 $\AA$ ). We measure the NH3360 index in our new data, and the Serven et al. (2005, SWB05 hereafter) optical indices in our SB06a optical spectra.

In SB03, we claimed that galaxies in the Coma cluster had much lower $\mathrm{CN}$ and $\mathrm{C} 4668$ indices than those in lower-density environments. We were unable then to conclude whether the differences were due exclusively to differences in carbon or if nitrogen was also overabundant in galaxies in lower-density environments. With the aim of resolving this issue, we selected our sample to include field, Virgo and Coma ellipticals, spanning a range in velocity dispersions $\left(130<\sigma<330 \mathrm{~km} \mathrm{~s}^{-1}\right)$. Following the nomenclature of SB03, we denote our Coma cluster sample as high-density environment galaxies (HDEG), while galaxies in the field and Virgo are denoted low-density environment galaxies (LDEG).

We have followed the standard reduction procedure for longslit spectra, using $\mathrm{RED}_{\mathrm{m}}^{\mathrm{uc}} \mathrm{E}$ (Cardiel 1999). Galaxy spectra were extracted within a central equivalent aperture of $4^{\prime \prime}$ at the distance of NGC 6703 (corresponding to a physical aperture of $0.76 \mathrm{kpc}$ ). We measured the NH3360 index using the definition of Davidge \& Clark $(1994)^{3}$ and the CNO3862, CNO4175, and CO4685 indices, as defined by SWB05. All indices were measured at a dispersion of $\sigma=200 \mathrm{~km} \mathrm{~s}^{-1}$ and corrected for the effects of velocity dispersion broadening using a combination of synthetic spectra from Bruzual \& Charlot (2003, BC03 hereafter) and A. Vazdekis et al. (2008, in preparation, V08 hereafter).

\section{RESULTS}

Figure 1 shows the co-added spectra in different wavelength regions of LDEG (blue) and HDEG (red), with velocity dispersions in the range of $150<\sigma<250 \mathrm{~km} \mathrm{~s}^{-1}$. Before being added, the spectra were shifted to the same radial velocity and broadened to the maximum $\sigma$ of all spectra $\left(250 \mathrm{~km} \mathrm{~s}^{-1}\right)$. The spectral regions correspond to the bands of four different indices: NH3360, CNO3862, CNO4175, and CO4685.

\footnotetext{
3 A case could be made to amend the Davidge \& Clark (1994) definition somewhat, in light of Carbon et al. (1994; Figure 13). Specifically, the NH3360 continua sit within the broad wings of the NH feature itself, reducing its optimal sensitivity to nitrogen. Redefining the red continuum to be 3480-3520 A would mitigate this effect to some degree, but for consistency with the canonical definition, we have not pursued this approach.
}
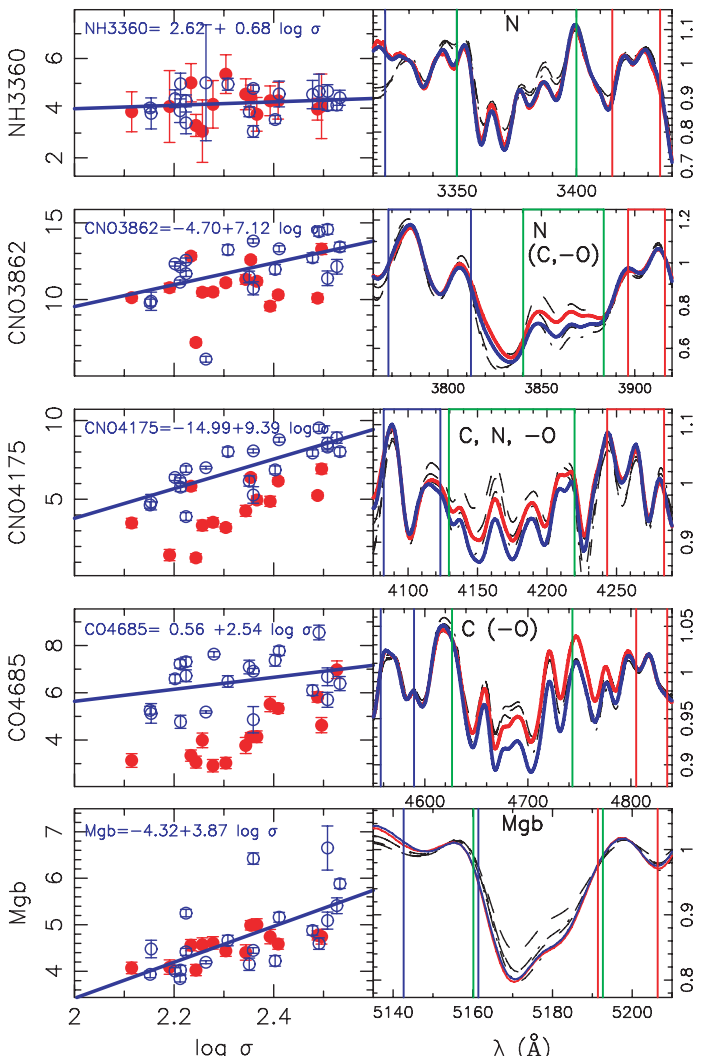

Figure 1. Left column: line-strength indices vs. velocity dispersion. Red solid circles correspond to HDEG, while blue open circles are LDEG. Right column: Blue, green, and red boxes represent the bandpass of the index definition: in blue and red are the two bands used to define the pseudocontinua. The flux in both the data and the models has been normalized to these bands. The central band, where the feature is located, is indicated in green. Black lines represent stellar population models. Only models with solar (dashed lines) and supersolar (dotted lines) metallicity are plotted; $6 \mathrm{Gyr}$ and $15 \mathrm{Gyr}$ models are plotted for each metallicity. The stacked spectra for LDEG are plotted in blue, while the HDEG are represented in red. Dominant species and other important species (within brackets) contributing to the indices defined by SWB05 are shown within the panels. A - symbol indicates that an increase in the abundance of that element makes the index weaker.

Overplotted in these diagrams are the models by $\mathrm{BC} 03$ for the $\mathrm{NH} 3360$ and the models of $\mathrm{V} 08$ for the remainder of the indices. We could not use V08 for the comparison of the NH index, because the spectra start at $3500 \AA$. However, we prefer to use it for the other regions of the spectra as the stellar library used in the $\mathrm{BC} 03$ models suffers from wavelength calibration problems. Nevertheless, we have checked and ensured that the results obtained are independent of the models employed. All synthetic spectra were degraded to the same broadened resolution as the galactic spectra. We have plotted four models in each figure, with ages $\sim 6$ and $15 \mathrm{Gyr}$, and solar and supersolar metallicities $([\mathrm{M} / \mathrm{H}]=+0.4$ for $\mathrm{BC} 03$ and $[\mathrm{M} / \mathrm{H}]=+0.2$ for $\mathrm{V} 08)$.

Comparing the empirical spectra with the models in Figure 1 and taking into account the element dependence of the indices 


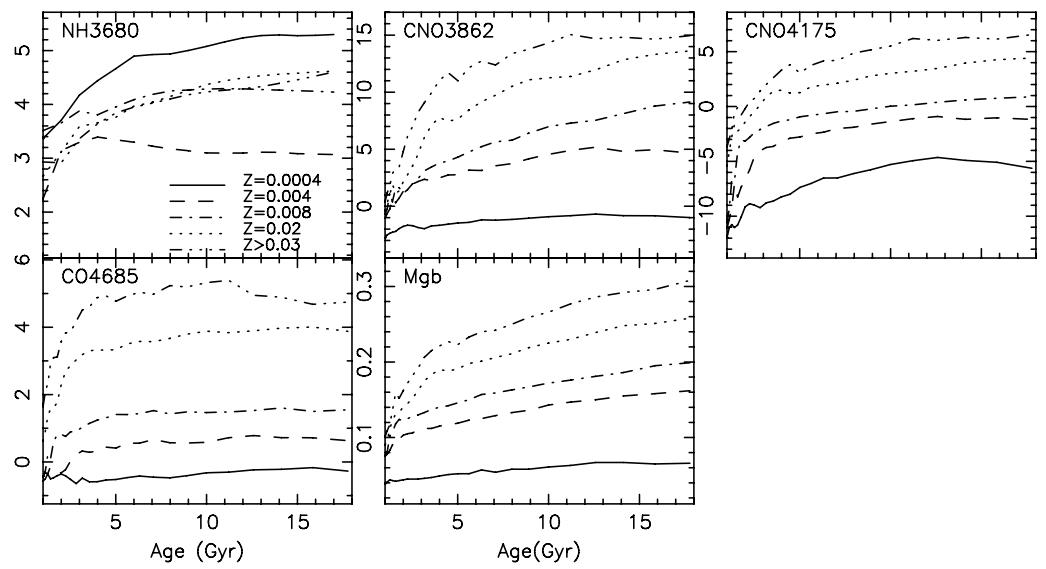

Figure 2. Variations of the analyzed indices with age at different metallicities as indicated. BC03 models are used for the NH3360, while V08 models are employed for the remainder of the plots.

(see the legend within the figure) we can see: NH3360 is slightly overabundant with respect to the scaled-solar models; CNO3862 is compatible with the scaled-solar models at lower metallicities (for older ages) or higher metallicities (for younger ages); CNO4175 and CO4682 are overabundant in the field galaxies, but not for the cluster galaxies. In contrast, these data require that nitrogen-based indices be overabundant with respect to solar, carbon-based indices be overabundant with respect to solar only for LDEG, and oxygen be similarly overabundant.

\subsection{The Index-Velocity Dispersion Relations}

In Figure 1, we present the behavior of the four indices analyzed, in addition to $\mathrm{Mgb}$, as a function of the velocity dispersion of the galaxies. We distinguish between three different groups of plots, depending on the chemical element dominating the intensity of the index: N-based indices (CNO3862 and NH3360), C-based indices (CNO4175 and CO4685), and Mgb. Indices dependent on $\mathrm{C}$ show a strong dependency upon velocity dispersion, in a similar way to $\mathrm{Mgb}$. However, the trends between the $\mathrm{N}$-sensitive indices and velocity dispersion are much flatter; indeed, they are even flatter than that found for $\langle\mathrm{Fe}\rangle$ (see SB03).

Differences in the slope of the index- $\sigma$ diagram could appear due to a different sensitivity of the indices to age and/or metallicity and do not necessarily reflect differences in the chemical abundance ratios. Figure 2 shows the variation of the indices with age for different metallicities. It can be seen that each of the SWB05 indices shows a strong dependence on metallicity, particularly CO4685. The Lick/IDS index Mgb also shows a strong metallicity dependence. On the other hand, for metallicities relevant for elliptical galaxies $(Z>0.004)$, NH3360 is essentially constant with age and overall metallicity. This behavior allows NH3360 to be used successfully to derive nitrogen abundances in integrated spectra, with Bessell \& Norris (1982) and Tomkin \& Lambert (1984) confirming its strong sensitivity to nitrogen abundance. ${ }^{4}$

Can we explain the relations between the other indices and $\sigma$ as the consequence only of a relation between the overall metallicity and/or age with $\sigma$ ? To answer this question, we have parameterized the models of $\mathrm{BC} 03$ and $\mathrm{V} 08$ as a function of age and metallicity, in the age and metallicity restricted to the range covered by our sample, $\sim 4-\sim 17$ Gyr and $-0.70<$

\footnotetext{
4 Note that the lack of metallicity dependence of the NH3360 index at $Z>0.04$ does not mean that this index is insensitive to $\mathrm{N}$ variations. In fact, the strong index measured in M31 globular clusters (Ponder et al. 1998) indicates that it is not.
}

$[\mathrm{M} / \mathrm{H}]<0.40$. If we allow for the same age variation with $\sigma$ (from $5 \mathrm{Gyr}$ to $12 \mathrm{Gyr}$ in the $\sigma$ range $125-350 \mathrm{~km} \mathrm{~s}^{-1}$ ) for all the indices, we need $\sim 0.13$ dex more metallicity variation to explain the $\mathrm{CNO} 4175$ - and $\mathrm{CO} 4685-\sigma$ relations - the most C-sensitive indices-while an extra $\sim 0.3$ dex variation in metallicity is needed to explain the relation of the $\mathrm{Mgb}-\sigma$ slope. At face value, this would indicate that $[\mathrm{C} / \mathrm{M}],[\mathrm{Mg} / \mathrm{M}]$ and $[\mathrm{Mg} / \mathrm{C}]$ increase with $\sigma$. The conclusions, however, remain very speculative until the oxygen abundance and its variation with $\sigma$ are calculated in these galaxies. In addition, the indices strongly dependent on $\mathrm{C}$ show a systematic offset between LDEG and HDEG, with the indices for HDEG systematically weaker than those for LDEG. ${ }^{5}$

\section{DISCUSSION}

We present here the NH3360 indices for a sample of 35 early-type galaxies. Unlike the ambiguities which exist when inferring nitrogen abundances from $\mathrm{CN}$ indices in the optical (due to contamination from carbon and oxygen in the bands and associated continua), the near-UV molecular feature of $\mathrm{NH}$ isolates the nitrogen abundance. The main conclusions of this work are: (1) a flat relation exists between the NH3360 index and the velocity dispersion of the galaxies, contrary to what has been previously claimed by other authors from their analysis of the more ambiguous $\mathrm{CN}$-index; (2) $\mathrm{N}$ is independent of local environment, whereas $\mathrm{C}$ appears stronger for LDEG relative to HDEG. In this sense, carbon and nitrogen production appear to be essentially decoupled from one another.

Our results can be useful for constraining the origin of $\mathrm{C}$ and $\mathrm{N}$. Nitrogen is produced in both intermediate-mass and massive stars, and it can have a primary and secondary origin (Carigi et al. 2005; Chiappini et al. 2006). The abundance ratio of a secondary element is predicted to increase with metallicity. If elliptical galaxies show a mass-metallicity relation, as has been claimed by many authors (e.g., Kodama \& Arimoto 1998), then we should expect the secondary $\mathrm{N}$ to increase with the mass of the galaxies, as suggested in previous works based upon $\mathrm{CN}$ inferred nitrogen abundance determinations (e.g., Kelson et al. 2006; Schiavon 2007). However, we have shown here that when a more "pure" nitrogen-sensitive indicator is used, there is no relation between the nitrogen abundance and the mass of the galaxies. This lack of correlation may be indicating primary production of nitrogen.

\footnotetext{
5 This behavior is also visible in the near-IR C-sensitive indices (E. Mármol-Queraltó 2009, in preparation).
} 
Nucleosynthesis studies predict that primary $\mathrm{N}$ can be produced both in the third dredge-up along the asymptotic giant branch (AGB) phase, if nuclear burning at the base of the convective envelope is efficient (Renzini \& Voli 1981, hot-bottom burning), and recent yields, including rotationally induced mixing, also predict significant production of $\mathrm{N}$ in massive stars (Meynet \& Maeder 2002). Chiappini et al. (2003, 2006) has shown that the inclusion of these yields in a Galactic chemical evolution model of the Milky Way predicts an N/O gradient consistent with observations. Having said that, this primary nitrogen production pathway is apparently only significant at low metallicities and is not predicted to be particularly relevant for systems such as elliptical galaxies that can reach supersolar metallicities very rapidly. In a system more similar to elliptical galaxies - that of the bulge of our galaxy-Ballero et al. (2007) (see also Matteucci 1986) need to include primary $\mathrm{N}$ from massive stars of all metallicities (and all masses) in order to explain the data from planetary nebulae.

In elliptical galaxies, the offsets between LDEG and HDEG favor massive stars as the main contributors to primary nitrogen, because for the elements produced by intermediate-mass stars, no offsets are detected. However, if that is the case, then we would expect a similar correlation between $\mathrm{N}$ and $\sigma$, as seen for $\mathrm{Mg}$ and $\sigma$. As we have shown, though, the $\mathrm{N}-\sigma$ correlation appears flat from our new data and analysis. A possible solution is that the contribution from both massive and intermediate-mass stars is shaping the $\mathrm{N}-\sigma$ relation in ellipticals. The delay in the $\mathrm{N}$ release from intermediate-mass $\left(4<M<8 M_{\odot}\right)$ stars is $\sim 100 \mathrm{Myr}$, so even in a relatively short star-formation burst, some contribution from these stars is expected. It is believed that the star formation in ellipticals is both more efficient and of a shorter timescale with increasing galactic mass. If this is the case, $\mathrm{N}$ would be produced increasingly more prominently by massive stars in the most massive galaxies, while the less massive galaxies with their more protracted starformation history would have an additional contribution from intermediate-mass stars. As mentioned, the delay in the release of $\mathrm{N}$ from intermediate-mass stars is $\sim 100 \mathrm{Myr}$ (the lifetime of 5-6 $M_{\odot}$ solar metallicity stars); intermediate-mass stars do not release significant amounts of $\mathrm{Mg}$ at high metallicities ${ }^{6}$, which would explain the different behavior of the $\mathrm{Mg}-\sigma$ relation compared with the $\mathrm{N}-\sigma$ relation.

Carbon is another controversial element. While some authors (e.g., Chiappini et al. 2003) argue that it is mainly produced in intermediate-mass stars (with masses between $1 M_{\odot}$ and 4 $M_{\odot}$ ), others suggest that massive stars are the main contributors (e.g., Carigi et al. 2005). The offset between galaxies in different environments that is not seen in other elements produced mainly in massive stars, such as $\mathrm{Mg}$, favors the suggestion that $\mathrm{C}$ is produced mainly in lower-mass stars. However, if low- and intermediate-mass stars are the main producers of $\mathrm{C}$, it is difficult to explain the strong correlation between $\mathrm{C}$ and $\sigma$ and the overabundance of $\mathrm{C} / \mathrm{Fe}$ with respect to the Sun. One possibility is that $\mathrm{C}$ is produced in both massive and lower-mass stars in comparable proportions. The significant slope in the relation would be the consequence of $\mathrm{C}$ production in massive stars, while the offset would come from a different production in lower-mass stars. This latter $\mathrm{C}$ release would increase the C budget in LDEG, while in the HDEG, star formation ceases before this can happen.

\footnotetext{
6 Note that significant contribution from AGB stars to the heavy isotopes ${ }^{25} \mathrm{Mg}$ and ${ }^{26} \mathrm{Mg}$ is predicted at metallicities lower than $[\mathrm{Fe} / \mathrm{H}]<-0.5$ (Fenner et al. 2003)
}

Of course, these are very simplified scenarios. The relative importance of the different $\mathrm{N}$ sites and the origin of $\mathrm{C}$ in elliptical galaxies will be explored in a future paper using our GEtool chemical evolution code (e.g., Hughes et al. 2008).

In summary, we report the first measurements of NH3360 for a large sample of elliptical galaxies covering a wide range of velocity dispersions. Contrary to what has been claimed previously, we do not find variations of the nitrogen abundance with the velocity dispersion of the galaxies. The environmental differences found for $\mathrm{C}$ and $\mathrm{N}$ abundances impose an important constraint on chemical evolution and galaxy formation models. It will be critical to independently constrain the oxygen abundance and its dependence upon galaxy mass, in part because of the obvious synergies between CNO in low- and intermediate-mass stars, and in part because oxygen is simply the most dominant component $(\sim 50 \%)$ of global metallicity.

We thank Francesca Matteucci and Reynier Peletier for several very useful discussions. The expert guidance of the referee, Judy Cohen, is gratefully acknowledged. This work has been partially supported by the Spanish research project AYA2007-67752-C03-03, the Marie Curie Intra-European Fellowship scheme within the 6th European Community Framework Programme (PSB), UK's Science \& Technology Facilities Council (BKG). E.T. acknowledges the support of the University of Central Lancashire's Livesey Award scheme (PSB).

\section{REFERENCES}

Ballero, S. K., Matteucci, F., Origlia, L., \& Rich, R. M. 2007, A\&A, 467, 123 Bessell, M. S., \& Norris, J. 1982, ApJ, 263, L29

Boulade, O., Rose, J. A., \& Vigroux, L. 1988, AJ, 96, 1319

Bruzual, G., \& Charlot, S. 2003, MNRAS, 344, 1000

Burstein, D. 2003, in ASP Conf. Ser. 297, Star Formation Through Time, ed. E. Perez, R. M. Gonzalez Delgado, \& G. Tenorio-Tagle (San Francisco, CA: ASP), 253

Burstein, D., Faber, S. M., Gaskell, C. M., \& Krumm, N. 1984, ApJ, 287, 586 Cardiel, N. 1999, PhD thesis, Univ. Complutense de Madrid, Spain

Carigi, L., Peimbert, M., Esteban, C., \& García-Rojas, J. 2005, ApJ, 623, 213 Chiappini, C., et al. 2006, A\&A, 449, L27

Chiappini, C., Romano, D., \& Matteucci, F. 2003, MNRAS, 339, 63

Clemens, M. S., et al. 2006, MNRAS, 370, 702

Davidge, T. J., \& Clark, C. C. 1994, AJ, 107, 946

Fenner, Y., et al. 2003, PASA, 20, 340

Graves, G. J., \& Schiavon, R. P. 2008, ApJS, 177, 446

Hughes, G. L., Gibson, B. K., Carigi, L., Sánchez-Blázquez, P., Chavez, J. M., \& Lambert, D. L. 2008, MNRAS, 390, 1710

Kelson, D. D., Illingworth, G. D., Franx, M., \& van Dokkum, P. G. 2006, ApJ, 653,159

Kodama, T., \& Arimoto, N. 1998, MNRAS, 300, 193

Matteucci, F. 1986, MNRAS, 221, 911

Meynet, G., \& Maeder, A. 2002, A\&A, 390, 561

Norris, J. E., Ryan, S. G., Beers, T. C., Aoki, W., \& Ando, H. 2002, ApJ, 569, L107

O’Connell, R. W. 1976, ApJ, 206, 370

Ponder, J. M., et al. 1998, AJ, 116, 2297

Renzini, A., \& Voli, M. 1981, A\&A, 94, 175

Sánchez-Blázquez, P., Gorgas, J., Cardiel, N., Cenarro, J., \& González, J. J. 2003, ApJ, 590, L91

Sánchez-Blázquez, P., Gorgas, J., Cardiel, N., \& González, J. J. 2006, A\&A, 457, 787

Schiavon, R. P. 2007, ApJS, 171, 146

Serven, J., Worthey, G., \& Briley, M. M. 2005, ApJ, 627, 754

Sneden, C. 1973, ApJ, 184, 839

Tomkin, J., \& Lambert, D. L. 1984, ApJ, 279, 220

Worthey, G. 1994, ApJS, 95, 107

Worthey, G. 1998, PASP, 110, 888 